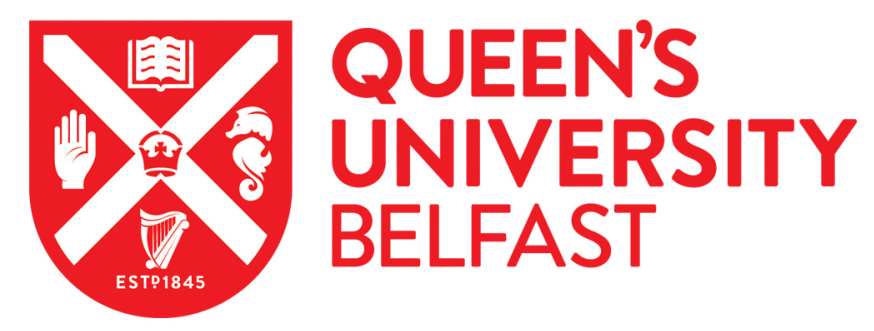

\title{
Auditory perception and speech discrimination after cochlear implantation in patients with connexin 26 (gjb2) gene-related Deafness
}

\author{
Sinnathuray, A. R., Toner, J. G., Geddis, A., Clarke-Lyttle, J., Patterson, C., \& Hughes, A. (2004). Auditory \\ perception and speech discrimination after cochlear implantation in patients with connexin 26 (gjb2) gene-related \\ Deafness. Otology \& Neurotology, 25(6), 930-934.
}

Published in:

Otology \& Neurotology

Queen's University Belfast - Research Portal:

Link to publication record in Queen's University Belfast Research Portal

\section{General rights}

Copyright for the publications made accessible via the Queen's University Belfast Research Portal is retained by the author(s) and / or other copyright owners and it is a condition of accessing these publications that users recognise and abide by the legal requirements associated with these rights.

Take down policy

The Research Portal is Queen's institutional repository that provides access to Queen's research output. Every effort has been made to ensure that content in the Research Portal does not infringe any person's rights, or applicable UK laws. If you discover content in the Research Portal that you believe breaches copyright or violates any law, please contact openaccess@qub.ac.uk. 


\title{
Auditory Perception and Speech Discrimination After Cochlear Implantation in Patients with Connexin 26 (GJB2) Gene-Related Deafness
}

\author{
*†Arasa Raj Sinnathuray, *Joseph G. Toner, *Andrea Geddis, \\ *Joanne Clarke-Lyttle, $\ddagger$ Christopher C. Patterson, and †Anne E. Hughes \\ *Northern Ireland Regional Cochlear Implant Center, Belfast City Hospital, Belfast, United Kingdom; \\ †Department of Medical Genetics, Queen's University, Belfast City Hospital, Belfast, United Kingdom; \\ and $\ddagger$ Department of Epidemiology \& Public Health, Queen’s University, Royal Victoria Hospital, \\ Belfast, United Kingdom
}

\begin{abstract}
Hypothesis: Auditory perception and speech discrimination among pediatric cochlear implantees may vary because of underlying deafness etiology, including connexin 26 (GJB2) gene-related deafness.

Background: Preliminary data suggest pathologic changes due to GJB2 mutations do not affect the spiral ganglion cells, which are stimulated by the cochlear implant. The survival of the spiral ganglion cells is believed to be an important determinant of outcome after surgery. Patients with GJB2-related deafness may therefore have enhanced prospects for good speech discrimination after implantation.

Methods: In an observational cohort study, GJB2 mutation analysis was performed using polymerase chain reaction amplification and direct sequencing on 31 prelingually deaf pediatric cochlear implantees, of which there were 30 with nonsyndromic deafness of unknown etiology, and one with keratitis-ichthyosis-deafness syndrome. Speech discrmination was assessed prospectively when they had reached postoperative year 3 using the IOWA Matrix Level B Sentences test and Glendonald Auditory Screening Procedure (GASP), with both patients and assessors blind to GJB2 status.
\end{abstract}

Results: Eleven patients had GJB2-related deafness and 20 patients had GJB2-unrelated deafness. IOWA Matrix scores were higher in patients with GJB2-related deafness but did not reach statistical significance. However, GASP scores were statistically significantly higher in patients with GJB2-related deafness (median word score, 92\%; median sentence score, $80 \%$ ), compared with those of patients with GJB2-unrelated deafness (median word score, 63\%; median sentence score, $45 \%$; word score, $p=0.037$; sentence score, $p=0.045$ ). Ordinal logistic regression analysis on IOWA Matrix and GASP sentence scores found better statistically significant scores in patients with $G J B 2$-related deafness $(p<0.05)$ after adjustment for confounding variables.

Conclusion: Pediatric cochlear implantees with GJB2-related deafness appear to have equal or better speech discrimination compared with a group of prelingually deaf children with deafness of unknown etiology. Key Words: Cochlear implantation-Connexin 26-Deafness-GJB2-Speech discrimination.

Otol Neurotol 25:930-934, 2004.
We have previously shown that children with GJB2related deafness, the most common known genetic cause of prelingual deafness (1), have significantly better speech intelligibility after cochlear implantation compared with those with deafness of unknown etiology (2). It would be interesting to investigate word recognition and higher levels of speech understanding in these children via outcome measures that require different levels of linguistic competence. Comparing these outcomes in implanted children, segregated according to GJB2 status,

Address correspondence and reprint requests to Dr. A.R. Sinnathuray, Research Fellow, Regional Cochlear Implant Center, Belfast City Hospital, Lisburn Road, Belfast BT9 7AB, United Kingdom; E-mail: rajsinn@aol.com may reveal where differences in their auditory systems exist and areas to be targeted by therapeutic advances toward improving speech intelligibility. Three small, single-center studies comparing prelingually deaf patients with cochlear implants and having GJB2-related deafness and GJB2-unrelated deafness have been reported (3-5). One found statistically better results in implanted patients with $G J B 2$-related deafness with regard to speech perception (6-mo follow-up, $\mathrm{N}=15, p=$ 0.0001) (4); however, no significant differences were found in the other two studies. We present a larger cohort study with longer follow-up of implanted patients, comparing auditory perception and speech discrimination in those with GJB2-related deafness to those with GJB2unrelated deafness. 


\section{MATERIALS AND METHODS}

\section{Recruitment of subjects and segregation into groups based on deafness etiologies}

This study considered 30 children with prelingual nonsyndromic deafness of unknown etiology (verified at interviews and ascertained from case notes) and one child with prelingual deafness due to keratitis-ichthyosis-deafness syndrome (6). All were in the Northern Ireland regional cochlear implant program and had undergone unilateral cochlear implantation by the same surgeon (J.G.T.) and had their devices activated for at least 3 years. Parents gave informed consent for genetic testing. The exclusion criteria were additional disorders with the potential to affect speech discrimination, such as developmental verbal dyspraxia (7).

All patients in our cohort were excluded as having had rubella virus embryopathy, cytomegalovirus embryopathy, measles, or mumps as the cause of their deafness, by history taking and by cross-referencing their names against data from the Regional Virology Laboratory. After genetic testing, children were grouped as having GJB2-related or GJB2-unrelated deafness. The demographic details of the children were evaluated after separation according to GJB2 status. Ethical approval was obtained from Queen's University Belfast Research Ethics Committee.

\section{GJB2 and GJB6 342 kb deletion mutation screening}

Methods of sampling, DNA extraction, and mutation screening have been previously described (2).

\section{Outcome measures}

Communication skills for the 31 children in this study were assessed at the fixed postoperative time interval of year 3 , before genetic testing commenced. Initially, we compared auditory perception using prospectively gathered implant-aided audiograms. These were assessed in the implanted ear and averaged over $500 \mathrm{~Hz}, 1 \mathrm{kHz}, 2 \mathrm{kHz}, 3 \mathrm{kHz}$, and $4 \mathrm{kHz}$ in all 31 cases. Speech discrimination results were gathered prospectively using the IOWA Matrix Level B closed-set sentence test $(8,9)$. Thirty information-carrying, four-word sentences were presented to the children, who responded by pointing to $4 \times 4$ picture matrices. For each word recognized correctly, each child was given a mark, giving a final score out of 120 . Results from prospectively gathered assessments using the Glendonald Auditory Screening Procedure (GASP) $(10,11)$ were also obtained. The GASP is an open-set speech discrimination test with two components of increasing difficulty. The word test, in which 12 words of different syllable number and/or stress pattern were spoken, was administered, and the children scored on how many they orally repeated correctly. The sentence test asked 10 questions with short answers and the children were scored on how many they orally responded to correctly. Raw scores in the IOWA Matrix and GASP tests were converted into percentages for ease of comparison, and both tests were administered in an auditory-only mode via live voice at approximately $70 \mathrm{~dB}$ sound pressure level. The tests were administered (usually by the same experienced member of the habilitation team) at the implant center, where the children were regular attendees.

Tests could not be performed by some children of very limited hearing ability; failure in easier tests clearly showed them to be too difficult. These children (four for the IOWA Matrix test, six for the GASP word test, and eight for the GASP sentence test) were accredited scores of 0 to avoid bias through exclusion (see Tables 1 and 2 for details). In an alternate situ-
TABLE 1. Individual scores on the IOWA Matrix Test and GASP word and sentence tests of children with GJB2-related deafness at postoperative year $3^{a}$

\begin{tabular}{cccc}
\hline & $\begin{array}{c}\text { Year 3 } \\
\text { IOWA } \\
\text { Matrix } \\
\text { Subject }\end{array}$ & $\begin{array}{c}\text { Year 3 } \\
\text { GASP } \\
\text { Word } \\
(\%)\end{array}$ & $\begin{array}{c}\text { Year 3 } \\
\text { GASP } \\
\text { Sentence } \\
(\%)\end{array}$ \\
\hline 5 & 95 & 92 & 100 \\
9 & 95 & 100 & 90 \\
14 & 92 & 100 & 90 \\
$10^{b}$ & - & 100 & 80 \\
2 & 100 & 92 & 80 \\
6 & 75 & 83 & 80 \\
8 & 87 & 92 & 60 \\
4 & 83 & 75 & 0 \\
$1^{c}$ & 98 & - & - \\
$7^{d}$ & 91 & - & $80(55-90)$ \\
$3^{d}$ & 66 & $92(79-100)$ & \\
Median & $91(81-96)$ & &
\end{tabular}

${ }^{a}$ Subject identification numbers are the same as those in our study on speech intelligibility (2).

${ }^{b}$ One child was not tested with IOWA Matrix test and was excluded because they were too tired.

${ }^{c}$ One child was not tested with GASP word and sentence tests but was included and accredited a score of zero (the tests were too difficult).

${ }^{d}$ Two children were not tested with GASP tests and were excluded because they were not cooperative.

ation, the IOWA Matrix test was not performed on two children and the GASP test was not performed on another two children because of tiredness or lack of cooperation; they had to be excluded. Hence, there is follow-up for 29 of 31 patients with regards to the IOWA Matrix test, and follow-up for a different subset of 29 of 31 patients with regards to the GASP word and sentence tests.

As a secondary outcome measure, we assessed at interview the amount of oral-only communication possible between parents and their implanted child at home at year 3 (i.e., without accompanying sign and/or gesture).

\section{Statistical analysis}

Demographic details and outcomes were compared as appropriate by the independent samples $t$ test, Mann-Whitney $U$ test, $\chi^{2}$ test, or Fisher's exact test. Ordinal logistic regression analyses (12) were performed to compare groups and to adjust for confounding variables. This approach requires categorization of outcome scores and assumes that the odds on a score being above a threshold for a category in one group relative to the other remains constant, irrespective of the choice of threshold in the outcome score, considered as the response variable. Tests of significance were conducted at the 5\% level $(p<0.05)$. Statistical analysis was performed using SPSS software version 11.0 (SPSS Inc., Chicago, IL, U.S.A.).

\section{RESULTS}

\section{GJB2-related deafness group $(\mathrm{N}=11)$}

Nine children had two pathogenic recessive mutations in GJB2: 7 homozygotes for $35 \mathrm{delG}$, and 2 compound heterozygotes for $35 \mathrm{delG} / 169 \mathrm{C}>\mathrm{T}$. A tenth patient carried a $35 \mathrm{delG}$ mutation in GJB2 and the $342 \mathrm{~kb}$ deletion in GJB6, a compound heterozygote for GJB2 and GJB6 
TABLE 2. Individual scores on the IOWA Matrix Test and GASP word and sentence tests of children with GJB2-unrelated deafness at postoperative year $3^{a}$

\begin{tabular}{|c|c|c|c|}
\hline Subject & $\begin{array}{c}\text { Year } 3 \\
\text { IOWA } \\
\text { Matrix } \\
(\%)\end{array}$ & $\begin{array}{c}\text { Year } 3 \\
\text { GASP } \\
\text { Word } \\
(\%)\end{array}$ & $\begin{array}{c}\text { Year } 3 \\
\text { GASP } \\
\text { Sentence } \\
(\%)\end{array}$ \\
\hline 24 & 93 & 100 & 100 \\
\hline 38 & 82 & 100 & 90 \\
\hline 36 & 100 & 92 & 90 \\
\hline 28 & 91 & 83 & 80 \\
\hline 39 & 79 & 75 & 70 \\
\hline $16^{b}$ & - & 92 & 60 \\
\hline 26 & 92 & 67 & 60 \\
\hline 23 & 91 & 67 & 60 \\
\hline 22 & 95 & 25 & 60 \\
\hline 21 & 92 & 42 & 50 \\
\hline 27 & 55 & 33 & 40 \\
\hline 25 & 74 & 92 & 20 \\
\hline 18 & 43 & 58 & 10 \\
\hline $30^{c, d}$ & 0 & 92 & 0 \\
\hline $29^{d}$ & 49 & 17 & 0 \\
\hline $20^{d, e}$ & 93 & 0 & 0 \\
\hline $19^{\mathrm{d}, \mathrm{e}}$ & 88 & 0 & 0 \\
\hline $15^{c-e}$ & 0 & 0 & 0 \\
\hline $17^{c-e}$ & 0 & 0 & 0 \\
\hline $31^{c-e}$ & 0 & 0 & 0 \\
\hline $\begin{array}{c}\text { Median } \\
\text { (interquartile range) }\end{array}$ & $82(43-92)$ & $63(4-92)$ & $45(0-68)$ \\
\hline
\end{tabular}

${ }^{a}$ Subject identification numbers are the same as those in our study on speech intelligibility (2).

${ }^{b}$ One child was not tested with IOWA Matrix test and was excluded because they were not cooperative.

${ }^{c}$ Four children were not tested with the IOWA Matrix test, but they were included and accredited a score of 0 since the test was too difficult.

${ }^{d}$ Seven children were not tested with GASP sentence tests, but they were included and accredited a score of 0 since the test was too difficult.

${ }^{e}$ Five children were not tested with the GASP word test, but they were included and accredited a score of 0 since the test was too difficult.

(13). The only syndromic child in this group had keratitis-ichthyosis-deafness syndrome caused by a de novo heterozygous dominant mutation in GJB2 (148G > A) that was absent in both parents (6). These 11 children were classified together as having GJB2-related deafness.

GJB2-unrelated deafness group $(\mathrm{N}=20)$

Eighteen children had neither GJB2 mutations nor GJB6 $342 \mathrm{~kb}$ deletions. A single recessive GJB2 mutation was identified in two children $(478 \mathrm{G}>\mathrm{A}$ and $249 \mathrm{C}>\mathrm{G})$. These 20 children were classified together as having GJB2-unrelated deafness.

\section{Demographic details}

All patients had preoperative, prelingual, profound deafness (preoperative unaided residual hearing thresholds $>90 \mathrm{~dB}$ hearing level) and received a full insertion of electrodes (all 22 active electrodes inside the cochlea) using the SPEAK coding strategy. In comparing those with GJB2-related deafness to those with GJB2unrelated deafness, there were no significant differences in the following variables: socioeconomic status, (manual: nonmanual) $6: 5$ versus 12:8 ( $p=1.00)$; sex, $(\mathrm{M}: \mathrm{F})$ 8:3 versus 13:7 ( $p=0.97)$; congenital onset of profound deafness, 11 of 11 versus 19 of 20 ( $p=1.00)$; preoperative aided auditory perception (mean $\mathrm{dB}$ hearing level [SD]) 65.2 [10.2] versus $65.9[10.1](p=0.85)$; contralateral hearing aid usage, 2 of 11 versus 1 of 20 ( $p=$ 0.28 ); timing of postoperative speech and language habilitation from start of program (median [range]) 3 years $[1-4 \mathrm{yr}]$ versus 3 years $[0-5 \mathrm{yr}](p=0.64)$; nucleus device/processor type, (CI22M/Spectra:CI24M/SPrint), $8: 3$ versus $16: 4(p=0.68)$; and implant daily usage amount during waking hours (median [range]) 14 hours [13-15 hr] versus 13 hours [9-16 hr] $(p=0.32)$.

In comparing those with GJB2-related deafness to those with GJB2-unrelated deafness, the following variables differed significantly: length of profound deafness (mean [SD]), 4.1 years [1.3 yr] versus 6.1 years [2.4 yr] $(p=0.02)$; age at implantation (median [range]) 3.9 years [2.5-6.3 yr] versus 5.8 years [2.8-10.3 yr] $(p=$ 0.007 ); and postoperative complications, 3 of 11 versus 0 of $20(p=0.04)$.

\section{Outcomes measures}

Children with GJB2-related deafness had better postoperative auditory perception (implant-aided thresholds, $500 \mathrm{~Hz}-4 \mathrm{kHz}$ ) than those with GJB2-unrelated deafness, but these differences were not statistically significant (Table 3). IOWA Matrix scores were also higher in those with GJB2-related deafness (median, 91\%) compared with those with GJB2-unrelated deafness (median, $82 \%)(p=0.09)$. However, GASP word and sentence scores differed significantly between the groups; patients with GJB2-related deafness (median word score, 92\%; median sentence score, $80 \%$ ) had statistically significantly higher scores than those with GJB2-unrelated deafness (median word score, 63\%; median sentence score, $45 \%$; word score, $p=0.037$; sentence score, $p=$ $0.045)$

Ordinal logistic regression analysis on categorized raw IOWA Matrix scores (0, 1-100, 101-110, and >110) and

TABLE 3. Comparisons of auditory perception and speech discrimination between patients with GJB2-related deafness and GJB2-unrelated deafness 3 years postoperatively

\begin{tabular}{|c|c|c|c|}
\hline & $\begin{array}{c}\text { GJB2-Related } \\
\text { Deafness }\end{array}$ & $\begin{array}{l}\text { GJB2-Unrelated } \\
\text { Deafness }\end{array}$ & Significance \\
\hline Year 3 postoperative aided auditory perception, mean dBHL (SD) & $39(3.7)$ & $41(4.8)$ & $p=0.18$ \\
\hline Year 3 GASP word test, median \% (interquartile range) & $92(79-100)$ & $63(4-92)$ & $p=0.037$ \\
\hline Year 3 GASP sentence test, median \% (interquartile range) & $80(55-90)$ & $45(0-68)$ & $p=0.045$ \\
\hline
\end{tabular}


categorized GASP scores (word: 0\%, 1-60\%, 61-90\%, 91-99\%, and 100\%; sentence: 0\%, 1-50\%, 51-60\%, 61$80 \%$, and $81-100 \%)$, considered age at implantation and socioeconomic status as confounders. As shown in Table 4 , the unadjusted odds ratio for higher postoperative IOWA Matrix scores in those with GJB2-related deafness compared with GJB2-unrelated deafness was 2.9 (95\% confidence interval $[\mathrm{CI}], 0.7-12.4 ; p=0.14$ ). After adjustment for the confounders, the odds ratio attained statistical significance: 6.8 (95\% CI, 1.2-38.3; $p=0.029$ ). The unadjusted odds ratio for higher postoperative GASP word scores in those with GJB2-related deafness compared with GJB2-unrelated deafness was 4.8 (95\% CI, 1.1-21.8; $p=0.04)$. The same comparison for postoperative GASP sentence scores was 4.2 (95\% CI, $1.0-18.4 ; p=0.057)$. After adjustment for the confounders, the odds ratio was no longer significant for GASP word scores at 3.4 (95\% CI, 0.7-17.0; $p=0.13$ ), but attained statistical significance for GASP sentence scores at 7.7 (95\% CI, 1.4-42.2; $p=0.019)$.

The amount of oral-only communication possible between parents and their implanted child at home was higher in families with children having GJB2-related deafness (100\% for all patients) compared with GJB2-unrelated deafness (median, 90\% [40-100\%]; $p=0.002$ ).

\section{DISCUSSION}

Because of age and language limitations, the IOWA Matrix Level B and GASP tests could usually only be administered from postoperative year 3 onwards. Year 3 also affords a reasonable compromise between availability of sufficient children for study and postoperative time, allowing genuine differences to emerge between the implanted groups with regard to all the outcome measures. The closed-set format in the IOWA Matrix test controls for learning effects associated with successive test presentations, making it a valid outcome measure to assess speech discrimination in young children with poor linguistic skills. Both the IOWA Matrix and GASP tests were assessed prospectively in our study, and are also widely used in other implant programs around the world (8-11). O'Donoghue et al. (9) found that by the third year postoperative interval, the median IOWA Matrix score in a cohort of 32 pediatric cochlear implantees was
$99 \%$. The medians of both groups in our study are lower than this, but O'Donoghue et al. had used the IOWA Matrix Level A closed-set sentence test, carried out in a similar format to the Level B test we used, but more appropriate for those with lesser linguistic skills. The GASP test was used by Waltzman et al. (11) to assess a cohort of 38 pediatric implantees preoperatively and postoperatively, revealing significant open-set speech recognition achieved after surgery.

A possible weakness in our study is that there were patients who had not actually performed the GASP or the IOWA Matrix tests and were included in analyses using accredited scores of zero, while others were excluded. Some children had limited abilities based on the results of easier tests, and were not assessed with the GASP or the IOWA Matrix tests because the habilitation team did not wish to have them feel a sense of failure. Accrediting them with scores of zero appropriately avoided bias through exclusion. Interestingly, these cases occurred more often in the GJB2-unrelated deafness group. Alternatively, four children who would not perform both tests because of tiredness or lack of cooperation were excluded from analyses of their "nonperformed" test. If these four had also been accredited with scores of zero in their "nonperformed" test, the differences in outcomes between groups may have narrowed, since three of the four were in the GJB2-related deafness group. It was, however, felt that accrediting them with a mark of zero may have underestimated their general known abilities, reflected partly by their results in the test they had performed (three of them scored at or above the group median score) (Tables 1 and 2).

The hearing advantage of children with GJB2-related deafness appears to be related to better speech understanding skills. These children performed better in all hearing outcome measures, although comparisons of postoperative audiograms and IOWA Matrix Level B scores did not reach statistical significance. Univariate analyses on GASP scores at year 3 did, however, reveal that children with GJB2-related deafness had significantly higher word and sentence scores than those with GJB2-unrelated deafness. There was also a significantly larger amount of oral-only communication possible between parents and their implanted child at home in families having children with GJB2-related deafness.

TABLE 4. Results of ordinal logistic regression on year 3 IOWA Matrix and GASP scores with adjustment for confounding variables

\begin{tabular}{|c|c|c|c|c|c|c|c|c|c|}
\hline \multirow[b]{2}{*}{ Scores adjusted for } & \multicolumn{3}{|c|}{ Year 3 IOWA Matrix Test } & \multicolumn{3}{|c|}{ Year 3 GASP Word Test } & \multicolumn{3}{|c|}{ Year 3 GASP Sentence Test } \\
\hline & $\begin{array}{l}\text { Odds } \\
\text { ratio }\end{array}$ & $95 \% \mathrm{CI}$ & $p$ & $\begin{array}{l}\text { Odds } \\
\text { ratio }\end{array}$ & $95 \%$ CI & $p$ & $\begin{array}{l}\text { Odds } \\
\text { ratio }\end{array}$ & $95 \% \mathrm{CI}$ & $p$ \\
\hline Unadjusted & 2.9 & $(0.7-12.4)$ & 0.14 & 4.8 & $(1.1-21.8)$ & 0.04 & 4.2 & $(1.0-18.4)$ & 0.057 \\
\hline Age at implantation & 7.0 & $(1.3-39.1)$ & 0.027 & 3.4 & $(0.7-17.0)$ & 0.13 & 7.4 & $(1.4-40.2)$ & 0.02 \\
\hline Socioeconomic status & 2.9 & $(0.7-12.3)$ & 0.14 & 4.9 & $(1.1-22.0)$ & 0.04 & 4.6 & $(1.0-20.6)$ & 0.04 \\
\hline $\begin{array}{l}\text { Socioeconomic status and } \\
\text { age at implantation }\end{array}$ & 6.8 & $(1.2-38.3)$ & 0.029 & 3.4 & $(0.7-17.0)$ & 0.13 & 7.7 & $(1.4-42.2)$ & 0.019 \\
\hline
\end{tabular}

The odds ratio estimates the odds of achieving a higher categorized year 3 IOWA Matrix score, year 3 GASP word score, or year 3 GASP sentence score in a patient having GJB2-related deafness relative to a patient having GJB2-unrelated deafness. 
Our numbers were large enough to use regressionbased analyses to adjust for potential confounders and to tease out the contribution of deafness etiology on outcome. For these analyses, categorization of IOWA Matrix and GASP scores was required. The resulting categories of IOWA Matrix and GASP scores were defined by ranges of values that allowed similar numbers within each category. Age at implantation and another known confounder, socioeconomic status, were controlled for in the regression analyses. Age at implantation was also one of the variables differing significantly between groups (lower in those with GJB2-related deafness).

The other two variables significantly different between groups; length of deafness and postoperative complications were not controlled for, since length of deafness was almost identical to age at implantation and postoperative complications were actually higher in those with GJB2-related deafness. In the regression analysis, the best outcomes were observed in those with GJB2-related deafness (Table 4). There was also a negative correlation noted between age at implantation and GASP word scores, but a significant positive correlation between age at implantation and IOWA Matrix test scores and between age at implantation and GASP Sentence scores. This could be because the latter two outcomes are more dependent on the linguistic vocabulary abilities of test subjects, which improves with age at testing, and those with later ages at implantation would be older at testing since they were compared at a fixed postoperative time interval of 3 years. Thus, despite longer auditory deprivation, a later age at implantation appeared to be a predictor of better outcome in the IOWA Matrix Level B test and GASP sentence test. Since those with GJB2-related deafness had the lowest ages of implantation, the odds ratios increased when adjusted for this in these two tests. This finding has also been shown in another study, where children implanted at an older age performed better at 2 years after implantation; however, the reverse occurred at the 3 and 4 year postoperative intervals (14). The variability of results in our study, depending on the outcome measure used, may reflect lack of sensitivity in these tests and/or may be related to the site(s) of pathologic changes in the auditory pathway of patients.

The findings of this study are that implanted children with GJB2-related deafness appear to have equal or bet- ter speech discrimination compared with a group of prelingually deaf children with deafness of unknown etiology.

Acknowledgments: We thank all participants of this study and Professor Quentin Summerfield, of the Medical Research Council Institute of Hearing Research in Nottingham, United Kingdom, for scientific and statistical advice.

We also thank Cochlear Europe Ltd. and the Mason Medical Research Foundation for making this study possible through research grants.

\section{REFERENCES}

1. Usami S, Koda E, Tsukamoto K, et al. Molecular diagnosis of deafness: impact of gene identification. Audiol Neurootol 2002;7: 185-90.

2. Sinnathuray AR, Toner JG, Clarke-Lyttle J, et al. Connexin 26 (GJB2) gene-related deafness and speech intelligibility after cochlear implantation. Otol Neurotol 2004;25:935-42.

3. Green GE, Scott DA, McDonald JM, et al. Performance of cochlear implant recipients with GJB2-related deafness. Am J Med Genet 2002;109:167-70.

4. Matsushiro N, Doi K, Fuse Y, et al. Successful cochlear implantation in prelingual profound deafness resulting from the common 233 delC mutation of the GJB2 gene in the Japanese. Laryngoscope 2002;112:255-61.

5. Fukushima K, Sugata K, Kasai N, et al. Better speech performance in cochlear implant patients with GJB2-related deafness. Int $J$ Pediatr Otorhinolaryngol 2002;62:151-17.

6. Richard G, Rouan F, Willoughby CE, et al. Missense mutations in GJB2 encoding connexin-26 cause the ectodermal dysplasia keratitis-ichthyosis-deafness syndrome. Am J Hum Genet 2002;70: 1341-8.

7. Groenen P, Maassen B, Crul T, et al. The specific relation between perception and production errors for place of articulation in developmental apraxia of speech. J Speech Hear Res 1996;39:468-82.

8. Tyler RS, Holstad B. A closed set speech perception test for hearingimpaired children. Iowa City: University of Iowa Department of Otolaryngology-Head and Neck Surgery, 1987.

9. O'Donoghue GM, Nikolopoulos TP, Archbold SM, et al. Speech perception in children after cochlear implantation. Am J Otol 1998; 19:762-7.

10. Erber NP. Auditory training. Washington, DC: A.G. Bell Association for the Deaf, 1982.

11. Waltzman SB, Cohen NL, Gomolin RH, et al. Open-set speech perception in congenitally deaf children using cochlear implants. Am J Otol 1997;18:342-9.

12. McCullagh P. Regression models for ordinal data. J R Statist Soc B 1980;42:109-42.

13. del Castillo I, Villamar M, Moreno-Pelayo MA, et al. A deletion involving the connexin 30 gene in nonsyndromic hearing impairment. N Engl J Med 2002;346:243-9.

14. Nikolopoulos TP, O'Donoghue GM, Archbold S. Age at implantation: its importance in pediatric cochlear implantation. Laryngoscope 1999;109:595-9. 